\title{
DESIGN OF INTERLEAVER WITH HIGH DIMENSIONAL ENCODING PRINCIPLE USING HYBRID GROUP SEARCH OPTIMIZATION
}

\author{
RUTUJA A. DESHMUKH ${ }^{1} \&$ DR. ASHISH R. PANAT ${ }^{2}$ \\ ${ }^{1}$ Research Scholar, Electronics Department, Priyadarshini College of Engineering, Nagpur, Maharashtra, India \\ ${ }^{2}$ Director Innovation, Incubation and Linkages, SNDT Women's University, Mumbai, Maharashtra, India
}

\begin{abstract}
Handling high dimensional data and an effective error correcting code with low complexity are the two main challenges in the communication system. In the previous paper [10], we have focused on the second problem of error correcting code and introduced a turbo encoder with an interleaver, which uses a hybrid meta-heuristic search algorithm by combining renowned Genetic Algorithm (GA) and Group Search Optimizer (GSO) in the name of hybrid GSO (HGSO) and that performs the high dimensional data transmission. Such a high dimensional data has transformed to low dimensional data by introducing a new high dimension interleaver in the conventional design in which dat a transformation and optimal pattern generation has taken place using the recent meta-heuristic search algorithm. The performance of the proposed system has compared with the existing GSO, Genetic Algorithm (GA), Firefly Algorithm (FA), Artificial Bee Colony (ABC) and random interleaver design in terms of signal to noise ratio (SNR), Bit Error Rate (BER) and Frame Error Ratio (FER) and also the computation time is computed for each component of both existing and proposed system. Finally, the experimental results show that the proposed high dimension interleaver design using HGSO approach performs well than any other methods.
\end{abstract}

KEYWORDS: Turbo Encoder, Interleaver, Hybrid GSO, SNR, BER, \& FER

Received: Jun 05, 2020; Accepted: Jun 25, 2020; Published: Oct 05, 2020; Paper Id.: IJMPERDJUN20201476

\section{INTRODUCTION}

Long term evolution (LTE) was elaborated by the third generation partnership project (3GPP) for the Wideband Code Division Multiple Access (WCDMA) based air interface [1]. LTE has a channel coding scheme called Turbo Coding. Parallel Concatenated Convolution Codes (PCCC) or Turbo codes are a strengthful species of channel codes that have a suboptimal iterative decoder whose performance near the capacity limit is extraordinary [2]. For turbo codes, an interleaver is a basic component, and its performance is based on its proper design. Interleaving is usually used in digital communication and storage systems to upgrade the performance of forward error correcting codes [1]. The basic structure of turbo code is designed as two recursive systematic convolutional (RSC) codes in parallel, and the second encoder receives an interleaved version of the original information sequence as input as shown in Fig. 1 [3]. Each RSC consists of memory registers (MR) and XOR gates. A codeword of turbo codes consists of the input bits to the first encoder followed by the parity bits and from both the constituent encoders and the performance of a turbo code can be evaluated by the means of bit error rate, i.e. the ratio of the number of inaccurately decoded bits to the number of all bits transmitted at certain time period.

In this circumstance, an interleaver is used to reduce the correlation between the parity bits corresponding to the original and interleaved data frames [3]. The interleaver is designed based on two major principles: (1) the 
distance spectrum properties reflecting the weight distribution of the code, and (2) the correlation between the soft output of the decoder corresponding to its parity bits and the information data sequence [3].

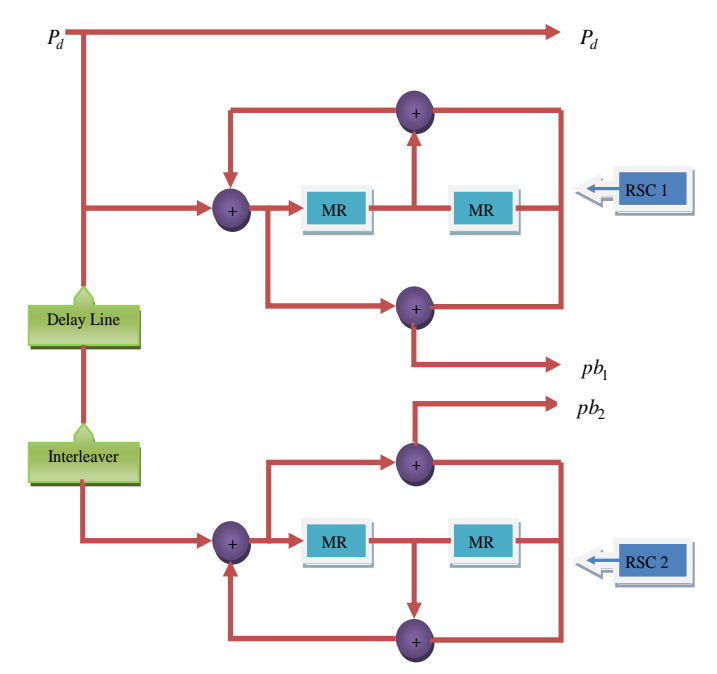

Figure 1: Simple Architecture of the Turbo Encoding System with an Interleaver.

Several algorithms [4] are designed to effectively evaluate free distances of large size interleavers with unconstrained input weight depends on constrained subcodes. The turbo code interleaver optimization is a nontrivial combinatorial task. Commonly, GA [5] is used for interleaver matrix optimization in which mutation and the crossover operator was omitted. The fitness criterion for every interleaver was maximum free distance. Another method which is frequently used for turbo code interleaver optimization is Differential Evolution (DE) as it works on real-valued vectors [6]. In [10], a robust interleaver design using hybrid meta-heuristic search algorithms is developed in which hybridization is performed between GSO and GA and that can be applied in high dimensional data transmission. In this paper, a high dimension interleaver is designed for data transformation process in which the high dimensional data has transformed to low dimensional data.

\section{LITERATURE REVIEW}

In 2015, Mingliang Zhang et al. [7] had presented a turbo coder in Quantum-dot cellular automata (QCA) to create error correction code (ECC). The aim of their work is to allow the new data to choose the paradigm based on a main-branch wire crossing (M-B) which is applicable to interleaver also. They approached a universal method for the implementation of feedback in IIR filters and also provide a two-stage pipelining interleaver. And finally, a turbo encoder is designed based on all these approaches, and the simulation demonstrates its performance

In 2016, Yassine Himeur et al. [8] proposed turbo coded-chaotic interleaving and frequency domain equalization scheme for high-speed OFDM-based power line communication systems (PLC). This method performs two stages of operation, in the first stage, a chaotic interleaving is employed for the turbo encoding and decoding process. In the second stage, frequency domain equalization is applied to ignore the noise samples in the frequency domain. The experimental result shows that this scheme has better performance than all other works in terms of BER, SNR, and security system.

In 2015, Shivani Pasricha et al. [1] had presented a Quadratic permutation polynomial (QPP) interleaver design which is stable for parallel turbo decoder implementation. VLSI architecture for the interleaver is analyzed in terms of overall delay, complete thermal power dissipation, total equivalent gate count and entire device utilization. Lastly, they 
presented a comparison between the proposed QPP interleaver and conventional interleaver.

In 2014, Prabhakar [9] had presented a solution to develop the free distance by optimizing turbo code interleaver using PSO and Genetic algorithms. They estimate the free distance based on the fitness function of PSO and GA. The performance of turbo codes can be improved by choosing the correct settings for the particular system. And the performance of PSO and GA is compared regarding free distance and Eb/NO Vs BER; they concluded that PSO performs well than GA and also the number of iterations essential for the convergence property in PSO is less when compared to GA.

In 2016, Rutuja and Ashish [10] has presented a hybrid meta-heuristic search algorithm by combining renowned Genetic Algorithm (GA) and GSO in the name of hybrid GSO (HGSO). The HGSO was emphasized to operate in high dimensional space so that the interleaver design was expected to be robust under high dimensional data transmission. The hybridization embodies the mutation operator of GA in the GSO scanning process. This has improved the exploration process of GSO to enable faster convergence. Experiments were conducted at higher order data bits and the performance of HGSO. A statistical report was prepared from the observed results to illustrate the reliability of the outcome accomplished by HGSO over the other methods.

\section{A. Problem Formulation}

In [10], we have introduced an interleaver design for the transformation of high dimensional data. The parameters of the design have optimally defined by the use of meta-heuristic search principle. In this paper, we are using a high dimension interleaver in which the data transformation and pattern generation have taken place, and the interleaver design utilizes another recent meta-heuristic search principle for the optimization process. The optimization process is carried out in two stages. In the first stage data transformation will take place, i.e., high dimensional data has transformed to low-dimensional data, and in the second stage, a hybrid meta- heuristic algorithm called HGSO is used to optimize the parameters of the interleaver design.

\section{PROPOSED INTERLEAVER DESIGN}

\section{B. Data Transformation}

The initial step of the proposed method can be accomplished by introducing a high dimension interleaver design in the architecture of turbo encoder scheme as shown in figure 2 to overcome the barrier of high dimensional data in turbo encoder which was illustrated in [10]. Commonly, an interleaver is used to preserve the performance of turbo encoding system. In this paper, an interleaver is designed along with an autoencoder to reduce the dimension of high data. Thus, the high dimensional data is transformed to low-dimensional data, and the transformed data is submitted for the optimal pattern generation. After the optimization process, the low dimensional patterned data is given to the recursive systematic convolutional (RSC 2) code of turbo encoder which encodes the received data as explained in [10]. 


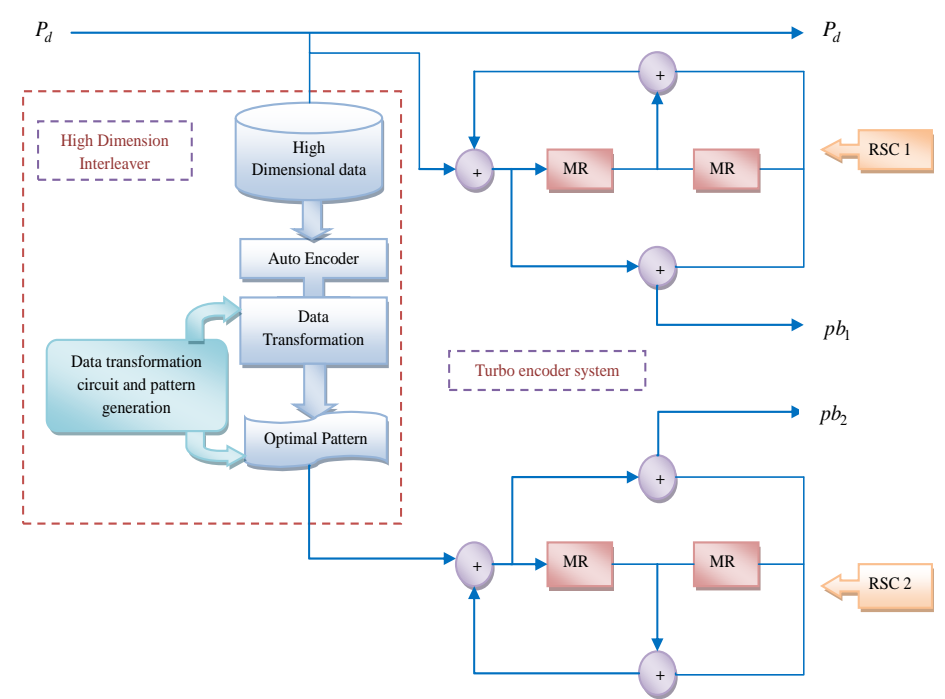

Figure 2: Proposed System Architecture of Turbo Encoder with a High Dimension Interleaver.

Let us explain the proposed system by considering an example for dimension reduction process of high dimension data. Consider a high dimension data with frame size, $N=1000$ with the solution length 10,000 . The main aim of proposed system is to maximize the distance between the data in terms of the cost function to reduce BER and SNR and also to reduce the computation time. The objective function is given by Eqn. (1).

$$
d^{*}=\arg \max _{d} F(d)
$$

The solution which obtained the maximum distance is referred as the best solution. In the example, the obtained best solution is given as $1 \times 10000$. The best solution is then submitted for reshaping process. Data reshaping is the process of reordering the contents of the data without altering the data contents. Thus the data has changed to the size $1000 \times 10$. Then, matrix multiplication has taken place between the actual frame size and the modified size as $[1 \times 1000] *[1000 \times 10]=[1 \times 10]$ thus the size of the data is reduced to $1 \times 10$. Here, the low dimensional data is obtained through the appropriate normalization and thresholding. In this technique, the $1 \times 10$ data is initially divided by the frame size $N$ to convert the data into 0's and 1's . Accordingly, the theresholding technique is applied (user defined threshold=0.25). If the resultant value is greater than 0.25 , then the value is assigned as 1 or else it is assigned as 0 . Then the final low dimensional and patterned data is given as input to the turbo encoding system.

\section{Hybridization of GA and GSO (Hybrid GSO or HGSO)}

After the data transformation process, the reduced data is submitted for optimal pattern generation using a meta-heuristic algorithm called HGSO which is employed in [10] that adopts the mutation operator of GA in the GSO. The scanning operation is the powerful key of the GSO for quick convergence. Thus, the product that is obtained from the zero left and right scanning is given in the following Eqn. (2), Eqn. (3) and Eqn. (4) in which the parent solution is denoted as $x_{p}$, the maximum pursuit angle is represented as $\theta_{\max }, l_{\max }$ is the maximum pursuit distance, $\varphi$ indicates the head angle, $D_{p}$ is the search direction, $r_{1}$ and $r_{2}$ are normally distributed and uniformly distributed random integer respectively.

$$
x_{\text {zero }}=x_{p}+r_{1} l_{\max } D_{p} \varphi(2)
$$




$$
\begin{aligned}
& x_{\text {left }}=x_{p}+r_{1} l_{\max } D_{p}\left(\varphi-r_{2} \theta_{\max } / 2\right) \\
& x_{\text {right }}=x_{p}+r_{1} l_{\max } D_{p}\left(\varphi+r_{2} \theta_{\max } / 2\right)
\end{aligned}
$$

Moreover, fine as well as broad searching can be enabled based on $r_{1}$. The zero scanning procedure can be modifying by HGSO using a simple mutation process adopted in GA. The hybrid GSO is derived by replacing the outcome of mutation $x_{m u t}$ with the outcome of zero scanning process $x_{z e r o}$ of GSO and is given in Eqn. (5) as,

$$
x_{m u t}=x_{\min }+\left(x_{\max }-x_{\min }\right) r_{1}
$$

We are using a simple mutation process to avoid the complexity of the operation in GSO.

The procedure that has to follow in HGSO model is shown in Fig. 3. In the first step, the process of encoding is carried out by initializing the solution $x_{q}: 0 \leq q \leq N_{p}-1$ which is obtained by producing an arbitrary integer set $[r]: r_{l} \in R ; 0 \leq l \leq L-1$ where $N_{p}$ refers to the number of possible solutions that is managed by HGSO and $L$ refers to the sum of information and tail bits and then arranging it in ascending order and achieving the index of the arranged order from the actual order. After the encoding process, each member of $x_{q}$ is used to evaluate the objective function using the Eqn. (6)

$$
I_{p}^{*}=\underset{\left\{I_{p}\right\}}{\arg \min } P_{b}\left(\hat{u}^{*}, u\right)
$$

In the above Eqn. (6), $I_{p}^{*}$ is the optimized interleaver pattern, $\left\{I_{p}\right\}$ is the set of potential interleaver patterns, $P_{b}(x, y)$ refers to the error probability between $x$ and $y$.

Secondly, the member whose objective function is minimum is selected as the producer, and that is denoted as $x_{p}$. In the final step, the operator has performed the operation that is similar to the GSO [11], except the zero scanning process, whereas, in HGSO, the zero scanning process utilizes the mutation operator given in Eqn. (5)

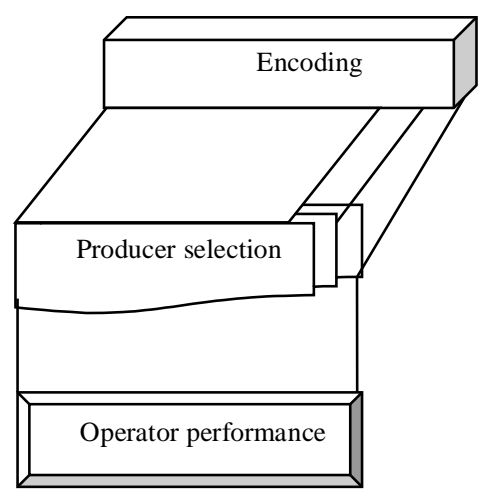

Figure 3: The Steps of Hybrid GSO Approach.

\section{Interleaver Design}

The design of an interleaver is already discussed in [10]. As stated in [10], the symbol error rate which is given in Eqn. (6) should be minimum. However, the sequence order is determined by maximum a posteriori (MAP) probability algorithm [12] 
[13] from Log-MAP decoder which is an optimal turbo decoder that is shown in figure 4

$$
\begin{aligned}
& \max ^{*}\left(x_{i}: 0 \leq i \leq n-1\right)=\ln \left[\sum_{i=0}^{n-1} e^{x_{i}}\right] \\
& \max ^{*}\left(x_{1}, x_{2}\right)=\max \left(x_{1}, x_{2}\right)+\ln \left(1+e^{-\left|x_{2}-x_{1}\right|}\right) \\
& \max ^{*}\left(x_{1}, x_{2}\right)=\max \left(x_{1}, x_{2}\right)+\ln \left(\left|x_{2}-x_{1}\right|\right) \\
& \max _{k}^{*}(x)=\max { }^{*}\left(x_{k-1}, x_{k}\right) \\
& \max ^{*}\left(x_{1}, x_{2}, x_{3}\right)=\max ^{*}\left(\max { }^{*}\left(x_{1}, x_{2}\right), x_{3}\right) \\
& \max _{k=3}^{*}(x)=\max { }^{*}\left(\max _{k=2}^{*}(x), x_{3}\right) \\
& \max _{k}^{*}(x)=\max { }^{*}\left[\max _{k-1}^{*}(x), x_{t}\right\rfloor \\
& \hat{u}^{*}=\underset{\hat{u}}{\arg \max P_{r}\{\hat{u} \mid y\}}
\end{aligned}
$$

Based on the eqn. (7) to eqn. (12), jacobian algorithm evaluates the logarithmic sum of exponential terms [14] as given in Eqn. (13). The most feasible sequence is obtained using the Eqn. (14).

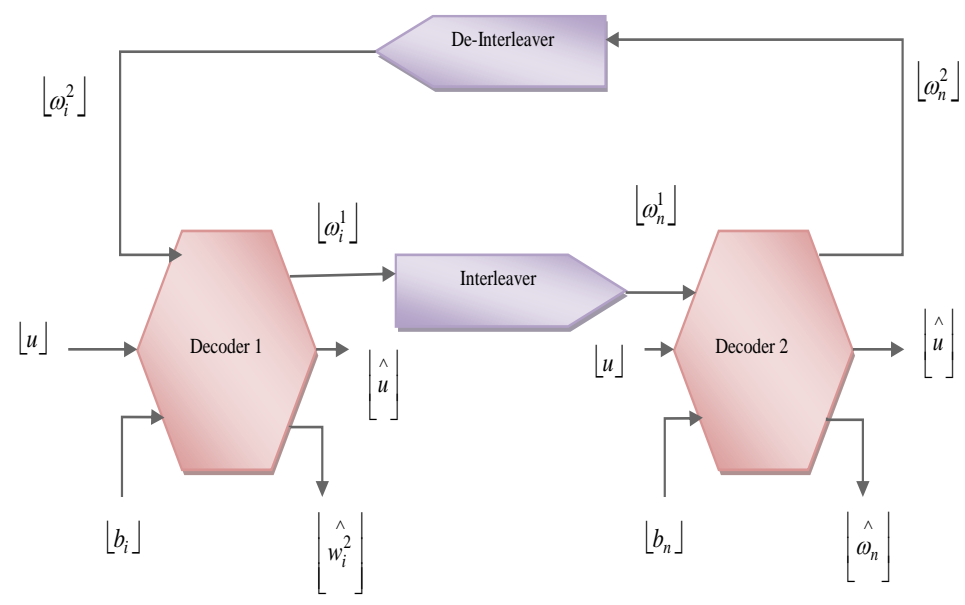

Figure 4: Architecture of Turbo Decoder with a High Dimension Interleaver.

\section{RESULTS AND DISCUSSIONS}

\section{E. Experimental Setup}

The simulation for designing an interleaver and its implementation are done in MATLAB R2015a. In this experiment, the classical turbo encoder - decoder system [15] is used as the base element. A high dimension interleaver is designed using HGSO, GSO, FA, ABC and GA and the results are compared with the performance of random interleaver. In [10], the optimized interleaver is developed with SNR at $0.1 \mathrm{Db}$ and its objective function is subjected to minimization, while in this paper the objective function is subjected to maximization and then the experimentation is carried out in two stages, in the 
first stage a high dimension interleaver is used and then the process is carried out in the turbo encoding system as shown in figure 2 for a high dimensional data with the data length 1000, 2000 and 3000. BER and FER against varying SNR have been noticed and the results are plotted in figure. 3 and figure. 4 respectively. The experiment is executed for ten times, and the results are tabulated as a statistical report in Table I, II, III, IV, V and VI.

\section{F. Discussion}

The statistical metrics such as best SNR, worst SNR, mean SNR, median SNR, Standard deviation SNR are reported using GSO, GA, FA, ABC, HGSO and random methods for the data with frames 1000, 2000 and 3000 are illustrated in table I, II and III. The time consumed by the interleaver and the encoder is computed for both conventional and proposed system, and it is tabulated in table IV, V and VI which is given below,

Table 1: Statistical Report on Various Interleaver Design Methods when Frame Size is Set to 1000 Bits

\begin{tabular}{|c|c|c|c|c|c|c|c|c|c|c|}
\hline \multirow{2}{*}{$\begin{array}{c}\text { Statistical } \\
\text { Metric }\end{array}$} & \multirow{2}{*}{ Methods } & \multicolumn{9}{|c|}{ (dB) } \\
\hline & & SNR1 & SNR2 & SNR3 & SNR4 & SNR5 & SNR6 & SNR7 & SNR8 & SNR9 \\
\hline \multirow{6}{*}{$\begin{array}{l}\text { Best } \\
\text { SNR }\end{array}$} & Random & 0.60126 & 0.0018712 & 0.0007696 & 0.00045768 & 0.0004399 & 0.00026126 & 0.00016916 & $7.2876 \mathrm{e}-05$ & $1.1 \mathrm{e}-06$ \\
\hline & GSO & 0.52418 & 0.0017759 & 0.00079758 & 0.00054032 & 0.00052255 & 0.00027778 & 0.00016438 & $7.9875 \mathrm{e}-05$ & $1.1 \mathrm{e}-06$ \\
\hline & GA & 0.58189 & 0.0016914 & 0.00075017 & 0.00043421 & 0.00041643 & 0.00020748 & 0.00011649 & $6.8876 \mathrm{e}-05$ & $1.1 \mathrm{e}-06$ \\
\hline & FA & 0.63741 & 0.001582 & 0.00073559 & 0.00051117 & 0.00049339 & 0.00027363 & 0.00017316 & $7.4876 \mathrm{e}-05$ & $1.1 \mathrm{e}-06$ \\
\hline & $\mathrm{ABC}$ & 0.58759 & 0.0015973 & 0.00082317 & 0.00051071 & 0.00049294 & 0.00028058 & 0.00014849 & $8.4875 \mathrm{e}-05$ & $1.1 \mathrm{e}-06$ \\
\hline & HGSO & 0.58451 & 0.0019882 & 0.0007761 & 0.00046014 & 0.00044237 & 0.0002744 & 0.00017394 & $7.6875 \mathrm{e}-05$ & $1.1 \mathrm{e}-06$ \\
\hline \multirow{6}{*}{$\begin{array}{l}\text { Worst } \\
\text { SNR }\end{array}$} & Random & 0.81326 & 0.81326 & 0.81326 & 0.81326 & 0.81326 & 0.81326 & 0.81326 & 0.81326 & 0.81326 \\
\hline & GSO & 0.58463 & 0.58463 & 0.58463 & 0.58463 & 0.58463 & 0.58463 & 0.58463 & 0.58463 & 0.58463 \\
\hline & GA & 0.83379 & 0.83379 & 0.83379 & 0.83379 & 0.83379 & 0.83379 & 0.83379 & 0.83379 & 0.83379 \\
\hline & FA & 0.80058 & 0.80058 & 0.80058 & 0.80058 & 0.80058 & 0.80058 & 0.80058 & 0.80058 & 0.80058 \\
\hline & $\mathrm{ABC}$ & 0.85217 & 0.85217 & 0.85217 & 0.85217 & 0.85217 & 0.85217 & 0.85217 & 0.85217 & 0.85217 \\
\hline & HGSO & 0.8069 & 0.8069 & 0.8069 & 0.8069 & 0.8069 & 0.8069 & 0.8069 & 0.8069 & 0.8069 \\
\hline \multirow{6}{*}{$\begin{array}{l}\text { Mean } \\
\text { SNR }\end{array}$} & Random & 0.72196 & 0.36231 & 0.24184 & 0.18151 & 0.14531 & 0.12114 & 0.10387 & 0.090895 & 0.080796 \\
\hline & GSO & 0.56642 & 0.28421 & 0.18978 & 0.14248 & 0.11409 & 0.095128 & 0.081567 & 0.071383 & 0.063452 \\
\hline & GA & 0.68994 & 0.34623 & 0.23108 & 0.17343 & 0.13884 & 0.11575 & 0.099234 & 0.086842 & 0.077193 \\
\hline & FA & 0.72574 & 0.364 & 0.24297 & 0.18236 & 0.146 & 0.12171 & 0.10435 & 0.09132 & 0.081174 \\
\hline & $\mathrm{ABC}$ & 0.75998 & 0.38162 & 0.25471 & 0.19117 & 0.15304 & 0.12759 & 0.10939 & 0.095728 & 0.085092 \\
\hline & HGSO & 0.70365 & 0.35319 & 0.23578 & 0.17698 & 0.1417 & 0.11814 & 0.10129 & 0.088639 & 0.078791 \\
\hline \multirow{6}{*}{$\begin{array}{l}\text { Median } \\
\text { SNR }\end{array}$} & Random & 0.72581 & 0.303 & 0.0022595 & 0.0014832 & 0.00086077 & 0.00068388 & 0.00056726 & 0.00053363 & 0.00047216 \\
\hline & GSO & 0.57576 & 0.26323 & 0.0019728 & 0.0014227 & 0.0009162 & 0.00069001 & 0.00056467 & 0.00054984 & 0.00054032 \\
\hline & GA & 0.67838 & 0.29283 & 0.0019509 & 0.0012702 & 0.00076527 & 0.00064744 & 0.00052693 & 0.00044625 & 0.00043147 \\
\hline & FA & 0.73991 & 0.32042 & 0.0020547 & 0.0013498 & 0.00086264 & 0.00066207 & 0.00056712 & 0.00054341 & 0.00051117 \\
\hline & $\mathrm{ABC}$ & 0.77845 & 0.29604 & 0.0022673 & 0.0014995 & 0.00089338 & 0.00069995 & 0.00060604 & 0.0005108 & 0.00048677 \\
\hline & HGSO & 0.69065 & 0.29588 & 0.0023174 & 0.0013604 & 0.00086219 & 0.00074982 & 0.00059922 & 0.00057983 & 0.00056045 \\
\hline \multirow{6}{*}{$\begin{array}{l}\text { STD_DEV } \\
\text { SNR }\end{array}$} & Random & 0.72581 & 0.303 & 0.0022595 & 0.0014832 & 0.00086077 & 0.00068388 & 0.00056726 & 0.00053363 & 0.00047216 \\
\hline & GSO & 0.57576 & 0.26323 & 0.0019728 & 0.0014227 & 0.0009162 & 0.00069001 & 0.00056467 & 0.00054984 & 0.0005403 \\
\hline & GA & 0.67838 & 0.29283 & 0.0019509 & 0.0012702 & 0.00076527 & 0.00064744 & 0.00052693 & 0.00044625 & 0.00043147 \\
\hline & FA & 0.73991 & 0.32042 & 0.0020547 & 0.0013498 & 0.00086264 & 0.00066207 & 0.00056712 & 0.00054341 & 0.00051117 \\
\hline & $\mathrm{ABC}$ & 0.77845 & 0.29604 & 0.0022673 & 0.0014995 & 0.00089338 & 0.00069995 & 0.00060604 & 0.0005108 & 0.00048677 \\
\hline & HGSO & 0.69065 & 0.29588 & 0.0023174 & 0.0013604 & 0.00086219 & 0.00074982 & 0.00059922 & 0.00057983 & 0.00056045 \\
\hline
\end{tabular}


Table 2: Statistical Report on Various Interleaver Design Methods when Frame Size is Set to 2000 Bits

\begin{tabular}{|c|c|c|c|c|c|c|c|c|c|c|}
\hline \multirow{2}{*}{$\begin{array}{c}\text { Statistical } \\
\text { Metric }\end{array}$} & \multirow{2}{*}{ Methods } & \multicolumn{9}{|c|}{ (dB) } \\
\hline & & SNR1 & SNR2 & SNR3 & SNR4 & SNR5 & SNR6 & SNR7 & SNR8 & SNR9 \\
\hline \multirow{6}{*}{$\begin{array}{l}\text { Best } \\
\text { SNR }\end{array}$} & Random & 0.63444 & 0.0016426 & 0.00061837 & 0.0004269 & 0.000409 & 0.0002416 & 0.00015694 & $7.6875 \mathrm{e}-05$ & $1.1 \mathrm{e}-06$ \\
\hline & GSO & 0.70907 & 0.0018154 & 0.00076822 & 0.00041671 & 0.00039894 & 0.00018093 & 0.00013105 & $7.9875 \mathrm{e}-05$ & $1.1 \mathrm{e}-06$ \\
\hline & GA & 0.62902 & 0.001448 & 0.0007828 & 0.00044901 & 0.00043124 & 0.00027427 & 0.00012005 & $6.8876 \mathrm{e}-05$ & $1.1 \mathrm{e}-06$ \\
\hline & FA & 0.56042 & 0.0015934 & 0.0008584 & 0.0005067 & 0.00048892 & 0.00022596 & 0.00012549 & $7.7875 \mathrm{e}-05$ & $1.1 \mathrm{e}-06$ \\
\hline & $\mathrm{ABC}$ & 0.58827 & 0.0021391 & 0.00088518 & 0.00050649 & 0.00048872 & 0.00029885 & 0.00019838 & 0.00010487 & $1.1 \mathrm{e}-06$ \\
\hline & HGSO & 0.58004 & 0.0017261 & 0.00083384 & 0.0004162 & 0.00039843 & 0.00023102 & 0.00014738 & $7.4876 \mathrm{e}-05$ & $1.1 \mathrm{e}-06$ \\
\hline \multirow{6}{*}{$\begin{array}{l}\text { Worst } \\
\text { SNR }\end{array}$} & Random & 0.87528 & 0.87528 & 0.87528 & 0.87528 & 0.87528 & 0.87528 & 0.87528 & 0.87528 & 0.87528 \\
\hline & GSO & 0.822 & 0.822 & 0.822 & 0.822 & 0.822 & 0.822 & 0.822 & 0.822 & 0.822 \\
\hline & GA & 0.79386 & 0.79386 & 0.79386 & 0.79386 & 0.79386 & 0.79386 & 0.79386 & 0.79386 & 0.79386 \\
\hline & FA & 0.76742 & 0.76742 & 0.76742 & 0.76742 & 0.76742 & 0.76742 & 0.76742 & 0.76742 & 0.76742 \\
\hline & $\mathrm{ABC}$ & 0.83383 & 0.83383 & 0.83383 & 0.83383 & 0.83383 & 0.83383 & 0.83383 & 0.83383 & 0.83383 \\
\hline & HGSO & 0.81542 & 0.81542 & 0.81542 & 0.81542 & 0.81542 & 0.81542 & 0.81542 & 0.81542 & 0.81542 \\
\hline \multirow{6}{*}{$\begin{array}{l}\text { Mean } \\
\text { SNR }\end{array}$} & Random & 0.73117 & 0.3669 & 0.24488 & 0.1838 & 0.14714 & 0.12267 & 0.10517 & 0.092037 & 0.081811 \\
\hline & GSO & 0.77706 & 0.3896 & 0.26004 & 0.19516 & 0.15623 & 0.13025 & 0.11167 & 0.097721 & 0.086863 \\
\hline & GA & 0.71779 & 0.35985 & 0.24019 & 0.18027 & 0.14432 & 0.12031 & 0.10315 & 0.090267 & 0.080237 \\
\hline & FA & 0.65933 & 0.33074 & 0.22086 & 0.16579 & 0.13274 & 0.11067 & 0.094891 & 0.083042 & 0.073816 \\
\hline & $\mathrm{ABC}$ & 0.7579 & 0.3805 & 0.25401 & 0.19065 & 0.15263 & 0.12725 & 0.1091 & 0.09548 & 0.084871 \\
\hline & HGSO & 0.71015 & 0.35606 & 0.23767 & 0.17839 & 0.14282 & 0.11907 & 0.10209 & 0.089341 & 0.079414 \\
\hline \multirow{6}{*}{$\begin{array}{l}\text { Median } \\
\text { SNR }\end{array}$} & Random & 0.72488 & 0.31955 & 0.0019627 & 0.0013298 & 0.00086444 & 0.00065672 & 0.00057635 & 0.00053962 & 0.00050305 \\
\hline & GSO & 0.81302 & 0.35579 & 0.0021554 & 0.0014512 & 0.00089305 & 0.0006846 & 0.00055782 & 0.00053347 & 0.00050911 \\
\hline & GA & 0.75826 & 0.31565 & 0.0019727 & 0.0011884 & 0.00089703 & 0.00067208 & 0.00054358 & 0.00052398 & 0.00045291 \\
\hline & FA & 0.62643 & 0.28148 & 0.0014288 & 0.0023975 & 0.0011654 & 0.00076122 & 0.0006186 & 0.00058179 & 0.0005067 \\
\hline & $\mathrm{ABC}$ & 0.79375 & 0.29628 & 0.0028097 & 0.0016648 & 0.00094681 & 0.00075554 & 0.00057936 & 0.00053531 & 0.00051594 \\
\hline & HGSO & 0.73415 & 0.29113 & 0.0019874 & 0.0013767 & 0.00091014 & 0.00075483 & 0.00059373 & 0.00056796 & 0.00048235 \\
\hline \multirow{6}{*}{$\begin{array}{l}\text { STD_DEV } \\
\text { SNR }\end{array}$} & Random & 0.72488 & 0.31955 & 0.0019627 & 0.0013298 & 0.00086444 & 0.00065672 & 0.00057635 & 0.00053962 & 0.00050305 \\
\hline & GSO & 0.81302 & 0.35579 & 0.0021554 & 0.0014512 & 0.00089305 & 0.0006846 & 0.00055782 & 0.00053347 & 0.00050911 \\
\hline & GA & 0.75826 & 0.31565 & 0.0019727 & 0.0011884 & 0.00089703 & 0.00067208 & 0.00054358 & 0.00052398 & 0.00045291 \\
\hline & FA & 0.62643 & 0.28148 & 0.0023975 & 0.0014288 & 0.0011654 & 0.00076122 & 0.0006186 & 0.00058179 & 0.0005067 \\
\hline & $\mathrm{ABC}$ & 0.79375 & 0.29628 & 0.0028097 & 0.0016648 & 0.00094681 & 0.00075554 & 0.00057936 & 0.00053531 & 0.00051594 \\
\hline & HGSO & 0.73415 & 0.29113 & 0.0019874 & 0.0013767 & 0.00091014 & 0.00075483 & 0.00059373 & 0.00056796 & 0.00048235 \\
\hline
\end{tabular}

Table 3: Statistical Report on Various Interleaver Design Methods when Frame Size is Set to 3000 Bits

\begin{tabular}{|c|c|c|c|c|c|c|c|c|c|c|}
\hline \multirow{2}{*}{$\begin{array}{c}\text { Statistical } \\
\text { Metric }\end{array}$} & \multirow{2}{*}{ Methods } & \multicolumn{9}{|c|}{$(\mathrm{dB})$} \\
\hline & & SNRI & SNR2 & SNR3 & SNR4 & SNR5 & SNR6 & SNR7 & SNR8 & SNR9 \\
\hline \multirow{6}{*}{$\begin{array}{l}\text { Best } \\
\text { SNR }\end{array}$} & Random & 0.62973 & 0.002124 & 0.00082572 & 0.00047424 & 0.00045646 & 0.00024409 & 0.00015627 & $9.0874 \mathrm{e}-05$ & $1.1 \mathrm{e}-06$ \\
\hline & GSO & 0.61065 & 0.0015943 & 0.00087467 & 0.00050543 & 0.00048765 & 0.00026405 & 0.0001446 & $7.3876 \mathrm{e}-05$ & $1.1 \mathrm{e}-06$ \\
\hline & GA & 0.53776 & 0.0016703 & 0.00082569 & 0.00049646 & 0.00047869 & 0.00026632 & 0.00014372 & $6.5876 \mathrm{e}-05$ & $1.1 \mathrm{e}-06$ \\
\hline & FA & 0.68832 & 0.0019039 & 0.00080963 & 0.00044036 & 0.00042258 & 0.00025518 & 0.00015471 & $7.2876 \mathrm{e}-05$ & $1.1 \mathrm{e}-06$ \\
\hline & $\mathrm{ABC}$ & & 0.0017783 & 0.00086774 & 0.00048711 & 0.00046933 & 0.00025697 & 0.00015649 & $7.6875 \mathrm{e}-05$ & $1.1 \mathrm{e}-06$ \\
\hline & HGSO & 0.54202 & 0.0020095 & 0.00086342 & 0.00052739 & 0.00050962 & 0.00027477 & 0.00015071 & $7.8875 \mathrm{e}-05$ & $1.1 \mathrm{e}-06$ \\
\hline \multirow{6}{*}{$\begin{array}{l}\text { Worst } \\
\text { SNR }\end{array}$} & Random & 0.78645 & 0.78645 & 0.78645 & 0.78645 & 0.78645 & 0.78645 & 0.78645 & 0.78645 & 0.78645 \\
\hline & GSO & 0.91695 & 0.91695 & 0.91695 & 0.91695 & 0.91695 & 0.91695 & 0.91695 & 0.91695 & 0.91695 \\
\hline & GA & 0.90932 & 0.90932 & 0.90932 & 0.90932 & 0.90932 & 0.90932 & 0.90932 & 0.90932 & 0.90932 \\
\hline & FA & 0.76084 & 0.76084 & 0.76084 & 0.76084 & 0.76084 & 0.76084 & 0.76084 & 0.76084 & 0.76084 \\
\hline & $\mathrm{ABC}$ & 0.80061 & 0.80061 & 0.80061 & 0.80061 & 0.80061 & 0.80061 & 0.80061 & 0.80061 & 0.80061 \\
\hline & HGSO & 0.70689 & 0.70689 & 0.70689 & 0.70689 & 0.70689 & 0.70689 & 0.70689 & 0.70689 & 0.70689 \\
\hline \multirow{6}{*}{$\begin{array}{l}\text { Mean } \\
\text { SNR }\end{array}$} & Random & 0.7198 & 0.36101 & 0.24099 & 0.18087 & 0.1448 & 0.12071 & 0.1035 & 0.090573 & 0.080509 \\
\hline & GSO & 0.75931 & 0.38067 & 0.25411 & 0.19074 & 0.15271 & 0.12731 & 0.10916 & 0.095527 & 0.084913 \\
\hline & GA & 0.71707 & 0.35976 & 0.24014 & 0.18024 & 0.1443 & 0.1203 & 0.10314 & 0.090256 & 0.080227 \\
\hline & FA & 0.72572 & 0.36419 & 0.24312 & 0.18248 & 0.14609 & 0.1218 & 0.10443 & 0.091389 & 0.081235 \\
\hline & $\mathrm{ABC}$ & 0.70746 & 0.35501 & 0.23701 & 0.1779 & 0.14243 & 0.11875 & 0.10181 & 0.089099 & 0.079199 \\
\hline & HGSO & 0.65639 & 0.32943 & 0.21995 & 0.16511 & 0.1322 & 0.11023 & 0.094508 & 0.082709 & 0.073519 \\
\hline \multirow{6}{*}{$\begin{array}{l}\text { Median } \\
\text { SNR }\end{array}$} & Random & 0.72818 & 0.31606 & 0.0022443 & 0.0015715 & 0.0009567 & 0.00070099 & 0.00055849 & 0.00051329 & 0.00047424 \\
\hline & GSO & 0.72094 & 0.30642 & 0.0021295 & 0.0013586 & 0.00095072 & 0.00082311 & 0.00059928 & 0.00057702 & 0.00054158 \\
\hline & GA & 0.74004 & 0.2713 & 0.0017656 & 0.0013364 & 0.00088464 & 0.00071174 & 0.00056511 & 0.00051862 & 0.00049646 \\
\hline & FA & 0.72497 & 0.34621 & 0.0023535 & 0.0015391 & 0.0010152 & 0.00075406 & 0.00059817 & 0.00056607 & 0.00048447 \\
\hline & $\mathrm{ABC}$ & 0.70944 & 0.27311 & 0.0024648 & 0.001465 & 0.0010078 & 0.00075421 & 0.00062261 & 0.00057161 & 0.00054968 \\
\hline & HGSO & 0.66753 & 0.27636 & 0.0021773 & 0.0015948 & 0.0010041 & 0.00075722 & 0.00061588 & 0.00057263 & 0.00054749 \\
\hline
\end{tabular}




\begin{tabular}{|c|c|c|c|c|c|c|c|c|c|c|}
\hline \multirow{6}{*}{$\begin{array}{l}\text { STD_DEV } \\
\text { SNR }\end{array}$} & Random & 0.72818 & 0.31606 & 0.0022443 & 0.0015715 & 0.0009567 & 0.00070099 & 0.00055849 & 0.00051329 & 0.00047424 \\
\hline & GSO & 0.72094 & 0.30642 & 0.0021295 & 0.0013586 & 0.00095072 & 0.00082311 & 0.00059928 & 0.00057702 & 0.00054158 \\
\hline & GA & 0.74004 & 0.2713 & 0.0017656 & 0.0013364 & 0.00088464 & 0.00071174 & 0.00056511 & 0.00051862 & 0.00049646 \\
\hline & FA & 0.72497 & 0.34621 & 0.0023535 & 0.0015391 & 0.0010152 & 0.00075406 & 0.00059817 & 0.00056607 & 0.00048447 \\
\hline & $\mathrm{ABC}$ & 0.70944 & 0.27311 & 0.0024648 & 0.001465 & 0.0010078 & 0.00075421 & 0.00062261 & 0.00057161 & 0.00054968 \\
\hline & HGSO & 0.66753 & 0.27636 & 0.0021773 & 0.0015948 & 0.0010041 & 0.00075722 & 0.00061588 & 0.00057263 & 0.00054749 \\
\hline
\end{tabular}

Table 4: Computational Time of Existing and Proposed Interleaver Design Methods when Frame Size is set to 1000 Bits

\begin{tabular}{|l|c|c|c|c|c|}
\hline \multirow{2}{*}{ Methods } & \multicolumn{2}{|c|}{ Existing } & \multicolumn{3}{c|}{ Proposed } \\
\cline { 2 - 6 } & Interleaver & Turbo encoder & Encoder & Interleaver & Turbo encoder \\
\hline GSO & 49.676 & 1058.4 & 26.643 & 3.394 & 23.846 \\
\hline GA & 75.896 & 955.6 & 2.7013 & 5.837 & 32.041 \\
\hline HGSO & 50.342 & 1067.4 & 19.479 & 3.9762 & 34.451 \\
\hline FA & 79.178 & 754.38 & 0.11365 & 5.2183 & 32.446 \\
\hline ABC & 80.726 & 1099.8 & 0.11819 & 5.8913 & 25.044 \\
\hline
\end{tabular}

Table 5: Computational Time of Existing and Proposed Interleaver Design Methods when Frame Size is Set to 2000 Bits

\begin{tabular}{|l|c|c|c|c|c|}
\hline \multirow{2}{*}{ Methods } & \multicolumn{2}{|c|}{ Existing } & \multicolumn{3}{c|}{ Proposed } \\
\cline { 2 - 6 } & Interleaver & Turbo encoder & Encoder & Interleaver & Turbo encoder \\
\hline GSO & 49.676 & 1058.4 & 54.567 & 2.7619 & 29.121 \\
\hline GA & 75.896 & 955.6 & 1.9845 & 4.9806 & 34.009 \\
\hline HGSO & 50.342 & 1067.4 & 93.034 & 3.2002 & 30.051 \\
\hline FA & 79.178 & 754.38 & 0.14305 & 8.8393 & 26.527 \\
\hline ABC & 80.726 & 1099.8 & 0.10801 & 8.1402 & 36.735 \\
\hline
\end{tabular}

Table 6: Computational time of Existing and Proposed Interleaver Design Methods when Frame Size is set to 3000 Bits

\begin{tabular}{|l|c|c|c|c|c|}
\hline \multirow{2}{*}{ Methods } & \multicolumn{2}{|c|}{ Existing } & \multicolumn{3}{c|}{ Proposed } \\
\cline { 2 - 6 } & Interleaver & Turbo Encoder & Encoder & Interleaver & Turbo Encoder \\
\hline GSO & 49.676 & 1058.4 & 107.91 & 300311 & 26.209 \\
\hline GA & 75.896 & 955.6 & 1.9468 & 4.6056 & 29.246 \\
\hline HGSO & 50.342 & 1067.4 & 69.734 & 3.1219 & 28.783 \\
\hline FA & 79.178 & 754.38 & 0.18085 & 4.3815 & 26.817 \\
\hline ABC & 80.726 & 1099.8 & 0.12025 & 5.7052 & 55.728 \\
\hline
\end{tabular}

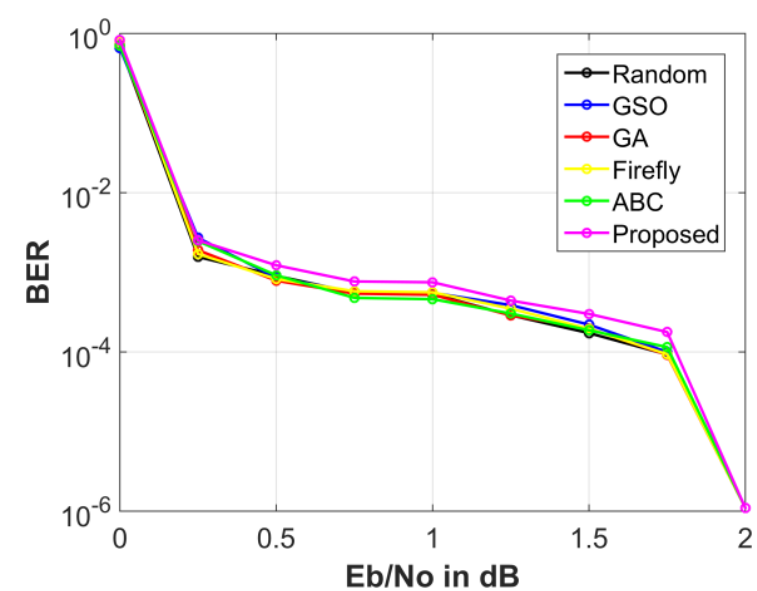

(a)

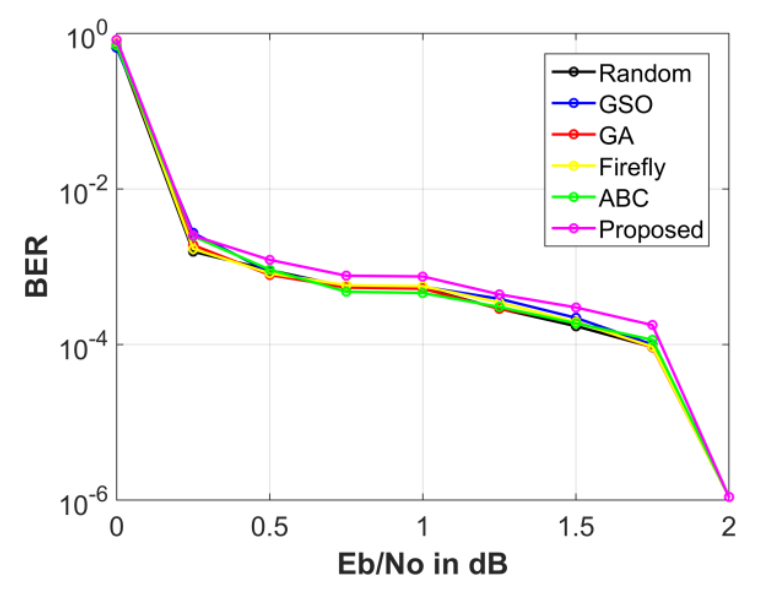

(b)

Figure 5: (a) BER Performance of Existing Method (b) BER Performance of Proposed Method. 


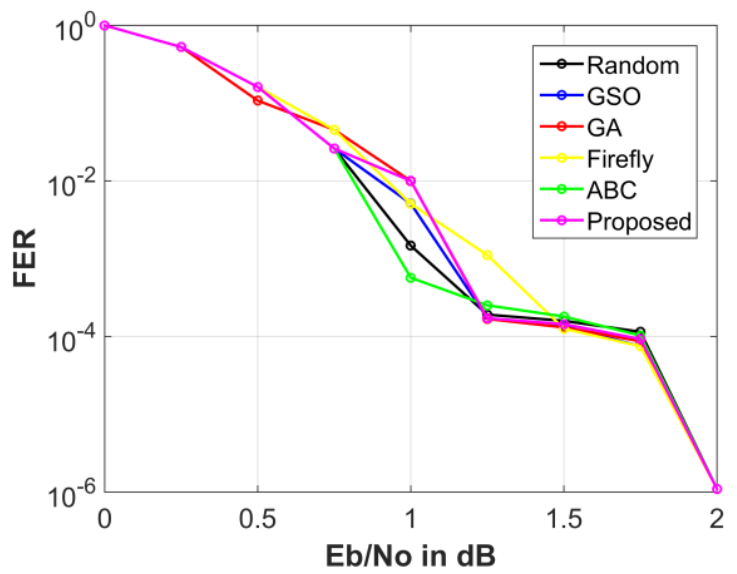

(a)

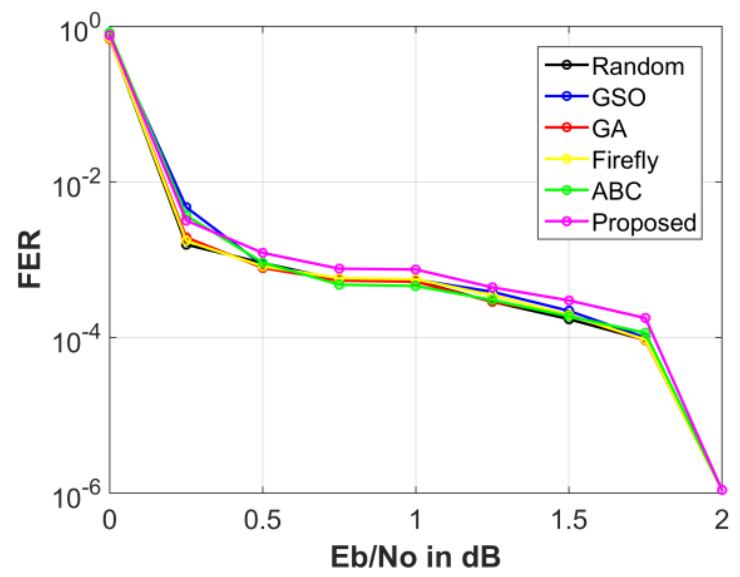

(b)

Figure 6: (a) FER Performance of Existing Method (b) FER Performance of Proposed Method.

Generally, the system performance can be improved by reducing the signal to noise ratio (SNR), bit error rate (BER) and frame error rate (FER). Here, the least signal to noise ratio (best SNR), maximum signal to noise ratio (worst SNR), mean SNR, median SNR, and standard deviation SNR are observed by executing a set of three data with a frame size 1000, 2000 and 3000 bits. Table I shows that the worst SNR maintains a constant signal to noise ratio of each variant for all the methods. However, on observing the statistical metrics, the performance of the proposed system can be concluded by stated that HGSO performance better than GSO except for the median SNR performance and GA overtake GSO in the median performance. It is found that among all the algorithms, the performance of random interleaver is very poor.

The statistical report for the data of frame size 2000 is illustrated in Table II, FA maintains a constant worst SNR throughout the execution, and in the overall execution of data with frame size 2000, HGSO possessed first position and GA acquired the last position. In Table III, the worst SNR of ABC method maintain its consistency throughout the execution, and it performs well in case of mean SNR when compare to all other algorithms. Random method slightly behaves better in the case of median SNR, but in all other metrics, its performance is poor. On taken the overall performance of data with frame size 3000 bits into account, HGSO secured first rank and random method secured the last rank. Thus from the final statistical report analyzation, it is clear that HGSO based high dimension interleaver is suitable for high dimensional data transmission.

In [10], we used two main components called an interleaver and turbo encoder for transferring high dimensional data. Now, we are using an extra encoder along with the conventional interleaver design in order to reduce the dimension of high volume data. Thus, the time required by each component of both conventional and proposed method for handling the data with the frame size 1000, 2000 and 3000 bits are computed and tabulated in Table IV, V and VI. On applying the algorithms GSO, GA, FA, ABC and HGSO on the existing and proposed methods, it is found that the components of proposed system consume less period for each frame size of data.

The figure 5 and figure 6 signify the BER and FER performance of both conventional and proposed methods, which shows that the bit error rate (BER) and frame error rate (FER) is reduced gradually in the proposed system than the existing system. Because of the reduction in error rate, the performance of the system gets improve throughout the execution. Thus, the overall experimental result proved that the new high dimension interleaver of the proposed system is working effectively with the turbo encoder by using HGSO approach. 


\section{CONCLUSIONS}

In the previous paper [10], we introduced a new hybrid GSO (HGSO), a Meta - heuristic search algorithm which was developed by hybridizing renowned GSO and GA in high dimensional space and so the turbo encoder exhibits high dimensional data transmission. While transferring such a high dimensional data, the system consumes more time, and the probability of both BER and FER is slightly high. So to overcome such a problem, a new high dimension interleaver is added with a turbo encoder scheme in the this paper. Data transformation and pattern generation are the two main works which are carried out in this interleaver, and a recent meta-heuristic search algorithm called HGSO is employed for the optimization process. The better performance of the proposed system has been experimentally proved by comparing with GSO, GA, FA, ABC and random interleaver design in case of its computation time, signal to noise ratio (SNR), BER and FER probabilities by HGSO than the other methods.

Since the BER minimization plays a major role in the turbo coding schemes, our research has attempted to consider it as the primary objective of interleaver design. However, interleaver design is also influenced by the spread and the girth of the interleaver. As this paper has produced encouraging results for meta-heuristic based interleaver design, the future research can be framed by considering the spread as well as maximizing girth as the secondary objectives. Hence, the problem model will become a multi-objective optimization problem and so the sophisticated optimization algorithms will be in a great need.

\section{REFERENCES}

1. Shivani Pasricha and Sanjay Sharma, “Novel Interleaver Design for Turbo Codes, ”vol. 86, no. 2, pp. 727-749, January 2016.

2. Massimiliano Laddomada and Bartolo Scanavino, “A Cost-Function Based Technique for Design of Good Prunable Interleavers for Turbo Codes, "' IEEE Transactions on Wireless Communications, vol. 5, no. 8, August 2006.

3. Muhammad Arif, Noor Muhammad Sheikh and Asrar U.H. Sheikh, "Design of two step deterministic interleaver for turbo codes, " Computers and Electrical Engineering, vol. 34, pp. 368-377, 2008.

4. R. Garello, F. Chiaraluce, P. Pierleoni, M. Scaloni and S. Benedetto, “On error floor and free distance of turbo codes, ” IEEE International Conference on Communications, vol. 1, pp. 45-49, 2001.

5. N. Durand, J. Alliot and B. Bartolome, "Turbo codes optimization using genetic algorithms," Proceedings of the Congress on Evolutionary Computation, vol. 2, pp. 816-822, 1999.

6. K. V. Price, R. M. Storn and J. A. Lampinen, "Differential Evolution A Practical Approach to Global Optimization," Natural Computing Series, Berlin.

7. Mingliang Zhang, Li Cai, Xiaokuo Yang, Huanqing Cui and Chaowen Feng, “Design And Simulation Of Turbo Encoder In Quantum-Dot Cellular Automata," IEEE Transactions on Nanotechnology, Vol. 14, No. 5, pp. 820-828, September 2015.

8. Yassine Himeur, Abdelkrim Boukabou and Abdelkader Senouci, "Performance of turbo-coded chaotic interleaving and frequency-domain equalization scheme for high speed OFDM-based PLC systems, ” Journal of the Franklin Institute, May 2016.

9. K. Prabhakar, Ch.Ravi Kumar and Dr.K.Padmaraju, “Interleaver Optimization By Particle Swarm Algorithm," International Journal For Technological Research In Engineering, vol. 2, no. 1, pp. 2347-4718, September 2014. 
10. Rutuja A. Deshmukh and Dr. Ashish R. Panat, "Hybrid Meta Heuristic Search Algorithm for High Dimensional Data Transmission", IJISET - International Journal of Innovative Science, Engineering \& Technology, vol. 3, no. 12, December 2016.

11. He, S.; Wu, Q.H.; Saunders, J.R., “Group Search Optimizer: An Optimization Algorithm Inspired by Animal Searching Behavior", IEEE Transactions on Evolutionary Computation, Vol: 13, No: 5, Page(s): 973 - 990, 2009.

12. P. Robertson, E. Villebrun, P. Hoeher, “A comparison of optimal and sub-optimal MAP decoding algorithms operating in the log domain, " Proc. IEEE International Conference on Communications (ICC), pp. 1009-1013, 1995.

13. P. Robertson, P. Hoeher, E. Villebrun, "Optimal and sub-optimal maximum a posteriori algorithms suitable for turbo decoding," pp. 119-125, 1997.

14. A.J. Viterbi, "An intuitive justification and a simplified implementation of the MAP decoder for convolutional codes," IEEE Journal on Selected Areas in Communications, vol. 16, no.2, pp. 260-264, 1998.

15. Won Y. Yang, Yong S. Cho, Won G. Jeon, Jeong W. Lee, Jong H. Paik, Jae K. Kim, Mi-Hyun Lee, Kyu I. Lee, Kyung W. Park, Kyung S. Woo, MATLAB®/Simulink® for Digital Communication, A-Jin Publishing Co., Korea, 2009. 